\title{
MULTI-MODALITY TOMOGRAPHIC IMAGE RECONSTRUCTION USING MESH MODELING ${ }^{1}$
}

\author{
Jovan G. Brankov, Yongyi Yang, Richard M. Leahy*, and Miles N. Wernick \\ Illinois Institute of Technology 3301 S. Dearborn St., Chicago, IL 60616 \\ *University of Southern California, 3740 McClintock Ave., Los Angeles, CA 90089
}

\begin{abstract}
In this paper we investigate a mesh-modeling approach for multi-modality image reconstruction. In the proposed approach a mesh model uses information obtained from an anatomical MR image to aid in reconstruction of PET images. The aim is to improve spatial resolution and quantitative accuracy of the PET image by using anatomical boundary information from the MR image. The mesh approach accomplishes this by using spatially adaptive spatial sampling and smoothing in the PET reconstruction. Our preliminary results demonstrate that this mesh-based approach to multi-modality PET reconstruction can achieve good results at low computational cost.
\end{abstract}

\section{INTRODUCTION}

In this paper we investigate a mesh-modeling approach for multi-modality image reconstruction. In particular, we consider the use of a mesh model to utilize information obtained from an anatomical magnetic resonance (MR) image to improve the reconstructed image quality from positron emission tomography (PET) data. Specifically, the goal is to improve spatial resolution and quantitative accuracy of the PET images, while respecting the differences that may exist between anatomical and functional image boundaries. Rather than imposing boundary information from the MR image onto the PET image, which may risk introducing false boundaries in the reconstruction, the proposed method uses spatially adaptive sampling and smoothing in an effort to allow, but not enforce, the development of edges.

In our previous work [1], a content-adaptive mesh modeling approach was proposed for two-dimensional (2D) image reconstruction. It was demonstrated that such an approach can outperform several well-known reconstruction algorithms in terms of both reconstructed image quality and computation time.

In a mesh model, the image domain is subdivided into a collection of mesh elements, the vertices of which are called nodes. The image function is then obtained over each element by interpolation from the values of these nodes [2]. In a content-adaptive mesh model (CAMM), the mesh elements are placed in a fashion that is adapted to the local content of the image. A mesh model of a $2 \mathrm{D}$ brain image [3] is shown in Fig. 1.

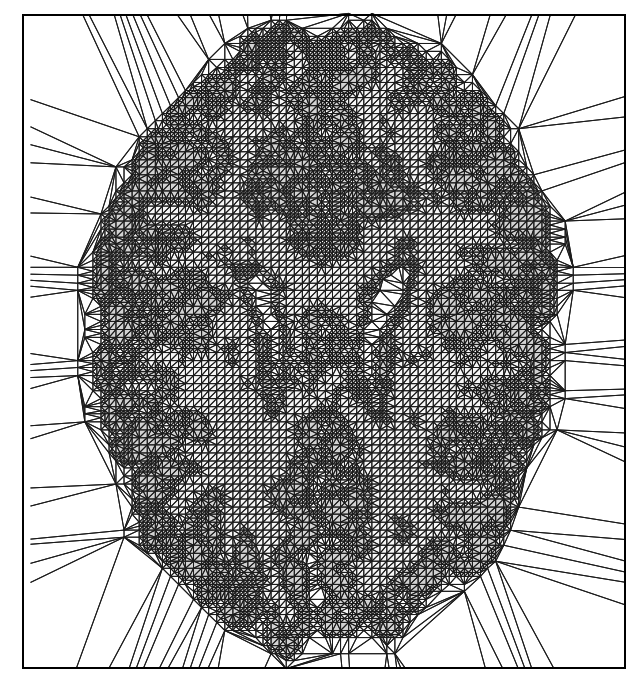

Figure 1. Mesh structure (8,887 nodes) obtained from segmented MR image.

In the proposed approach, a CAMM is first established based on an anatomical MR image represented on a fine pixel grid. This mesh model serves as the basis for a customized basis representation of the image. The parameters of this image representation are then estimated from the PET data.

Pixel-based methods have been proposed before for incorporating $\mathrm{MR}$ anatomical priors to improve PET image reconstruction [4-8]. In [5], for example, a prior distribution was explicitly defined to incorporate the anatomical data in a Bayesian framework. Our proposed approach aims to achieve the same objective, but does so by means of a content-adaptive mesh structure. In addition to the potential image-quality advantage of the mesh approach, it provides a compact image representation

\footnotetext{
${ }^{1}$ This research was supported by the Whitaker Foundation and by NIH/NHLBI grant HL65425.
} 
(having fewer unknowns), which can alleviate the underdetermined nature of the reconstruction problem and the data storage requirement, and can also lead to a fast computation.

\section{MODEL DESCIPRTION}

\subsection{Mesh representation model}

Let $f(\mathbf{x})$ denote the image function defined over a domain $D$. In a mesh model, the domain $D$ is partitioned into $M$ non-overlapping mesh elements (here triangular), denoted by $D_{m}, m=1,2, \cdots, M$. The image function is represented as

$$
f(\mathbf{x})=\sum_{n=1}^{N} f\left(\mathbf{x}_{n}\right) \varphi_{n}(\mathbf{x})+e(\mathbf{x}),
$$

where $\mathbf{x}_{n}$ is the $n$th mesh node, $\varphi_{n}(\mathbf{x})$ is a linear interpolation basis function associated with $\mathbf{x}_{n}, N$ is the total number of mesh nodes used, and $e(\mathbf{x})$ is the modeling error. Now let $\mathbf{n}$ denote a vector formed by the nodal values of the mesh model, i.e.,

$$
\mathbf{n} \equiv\left[f\left(\mathbf{x}_{1}\right), f\left(\mathbf{x}_{2}\right), \cdots f\left(\mathbf{x}_{n}\right)\right]^{T} .
$$

If $\mathbf{f}$ denotes the voxel representation of the image function $f(\mathbf{x})$ over $D$, then from (1) and (2) one can obtain

$$
\mathbf{f}=\Phi \mathbf{n}+\mathbf{e}
$$

where $\Phi$ is an interpolation matrix formed from $\varphi_{n}(\mathbf{x})$ in (1), and $\mathbf{e}$ is a vector representing the error $e(\mathbf{x})$.

\subsection{Dual-modality mesh generation}

Here we consider the use of an MR image for improved reconstruction of PET images. We hypothesize that this may allow better reconstruction of boundaries in the PET image by utilizing, but not enforcing, MR boundary information. This is accomplished in part by increasing the spatial sampling rate of the PET image near anatomical image boundaries identified from the MR image.

To obtain the mesh structure from the MR brain image we employ the following steps. First, a 256x256 MR image is segmented into three pixel types: grey matter (GM), white matter (WM), and cerebrospinal fluid (CSF), by a procedure described in [3]. Second, these segmented regions are assigned different gray levels: 0 for CSF, 1 for WM, 2 for GM, and 1 for the region outside the brain for reasons having to do with the mesh-generation algorithm used (described in our previous work [9]). Third, this mesh-generation algorithm is appplied to place the mesh nodes automatically in such a way that they are arranged densely along the anatomical boundaries, but with few nodes elsewhere. Finally, additional mesh nodes are placed in the interior of all the brain regions, using a lower-resolution $(128 \times 128)$ sampling pattern than the MR grid (256x256). This is in consideration of the fact that the PET image is expected to contain functional variations within image regions that are uniform in the segmented MR image. The resulting mesh is shown in Fig. 1.

\subsection{Mesh reconstruction model}

For tomographic image reconstruction, the imaging equation is typically written in terms of the pixel representation $\mathbf{f}$ as

$$
E[\mathbf{g}]=\mathbf{H f},
$$

where $\mathbf{g}$ contains the measured data, $E[\cdot]$ is the expectation operator, and $\mathbf{H}$ is a matrix describing the imaging system response.

Substituting (3) into (4), we obtain the mesh-domain imaging equation:

$$
E[\mathbf{g}]=\mathbf{H}[\Phi \mathbf{n}+\mathbf{e}] \equiv \mathbf{A n}+\hat{\mathbf{e}},
$$

where $\mathbf{A}=\mathbf{H} \Phi$ and $\hat{\mathbf{e}}=\mathbf{H e}$.

The modeling error $\hat{\mathbf{e}}$ of the basis representation in (5) is negligible in comparison with the noise level in PET imaging data. Thus, we obtain

$$
E[\mathbf{g}] \approx \mathbf{A n} .
$$

The reconstruction problem becomes one of estimating the nodal values in $\mathbf{n}$ from the observed data in $\mathbf{g}$. The image $\mathbf{f}$ can then be obtained from (3) (neglecting $\mathbf{e}$ ).

\subsection{Statistical image reconstruction}

In our study we used a maximum a posteriori (MAP) estimate of the nodal values in $\mathbf{n}$, which is obtained as

$$
\hat{\mathbf{n}}=\arg \max _{\mathbf{n}}[\log p(\mathbf{g} ; \mathbf{n})+\log p(\mathbf{n})],
$$

where $p(\mathbf{g} ; \mathbf{n})$ is the likelihood function of $\mathbf{g}$ parameterized by $\mathbf{n}$, and $p(\mathbf{n})$ is a prior on the unknown nodal values. We assume a Poisson likelihood, which is characteristic of emission tomography. The prior $p(\mathbf{n})$ is described by the Gibbs distribution [4], i.e.,

$$
p(\mathbf{n}) \sim \exp (-\beta \mathrm{U}(\mathbf{n}))
$$

where $\beta$ is a scalar weighting parameter, and $\mathrm{U}(\mathbf{n})$ is the energy sum of individual nodal values:

$$
\mathrm{U}(\mathbf{n})=\sum_{n=1}^{N} \sum_{j \in \Re_{n}} w_{n, j}\left(\mathrm{n}_{\mathrm{j}}-\mathrm{n}_{\mathrm{n}}\right)^{2} \text {. }
$$

In (8), $\mathfrak{R}_{n}$ denotes the set of nodes connected to the $\mathrm{n}^{\text {th }}$ node, and $w_{n, j}$ are weighting factors that can be chosen to be adaptive to the mesh structure.

The MAP estimate in (7) can be computed by using the following one-step-late expectation-maximization (OSLEM) algorithm [10,11]: 


$$
\mathrm{n}_{s}^{\text {new }}=\frac{\mathrm{n}_{s}^{\text {old }}}{\sum_{t} \mathrm{~A}_{t s}+\beta \frac{\partial}{\partial \mathrm{n}_{s}^{\text {old }}} \mathrm{U}\left(\mathbf{n}^{\text {old }}\right)} \sum_{t} \mathrm{~A}_{t s}\left(\frac{\mathrm{g}_{t}}{\sum_{k} \mathrm{~A}_{t k} \mathrm{n}_{k}^{\text {old }}}\right),(10)
$$

where $\mathrm{n}_{s}^{\text {old }}$ is the value of node $s$ from the previous iteration, $\mathbf{g}_{t}$ is the recorded count for observation $t$, and $\mathbf{A}_{t s}$ is the $t s$ entry of matrix $\mathbf{A}$.

\section{EXERIMENTAL RESULTS}

\subsection{Evaluation data}

In our experiment a single slice from an anatomical MRI brain scan was used. The image consists of 256x256 pixels of dimension $1 \mathrm{~mm}$ (Fig. 2 (left)). The MR image was segmented into three pixel types: grey matter (GM), white matter (WM), and cerebrospinal fluid (CSF) by a procedure described in [3].

The segmented MR image was then used to generate a phantom for PET simulation using relative activity levels of 4:1:0 for GM, WM, and CSF, respectively. The resulting phantom is shown in Fig.2 (right). A 128x128 sinogram was simulated, at a level of $1 \mathrm{M}$ counts, using intrinsic resolution of $3 \mathrm{~mm}$ full width at half-maximum (FWHM).

In addition, a mesh structure (shown in Fig.1) was obtained using the procedure described in Section 2.2 from the segmented MR image. This mesh strurcture was then used to reconstruct the PET images.

\subsection{Reconstruction methods considered}

The proposed method was studied with the following choices for the prior: 1) no prior assumed (MESH ML); 2) a quadratic Gibbs prior with $w_{n, j}=1$ in (9) (MESH MAP); 3) a quadratic Gibbs prior with $w_{n, j}$ set to $1 / 5$ when $\mathrm{n}_{\mathrm{j}}$ and $\mathrm{n}_{\mathrm{n}}$ are adjacent pixels, and 1 otherwise (MESH $M A P-W)$.

For comparison, the following pixel-based methods were also considered: 1) a maximum-likelihood EM algorithm (Pixel ML); 2) OSL-MAP reconstruction with a quadratic Gibbs prior (Pixel MAP).

Post-filtering was applied to both the pixel-based ML and mesh-based ML methods. All the iterative reconstruction algorithams were run for 50 iterarions.

\subsection{Evaluation criteria}

To evaluate the performance of the proposed algorithms, bias-variance curves were computed for both the WM and GM regions in a selected region of interest of the phantom, indicated in white in Fig.2 (right).

\subsection{Simulation results}

For visual comparison, we show in Fig. 3 some images obtained from the reconstruction methods tested. The MESH MAP-W method (i.e., spatially-varying prior) appears to produce slightly better images than the other methods.
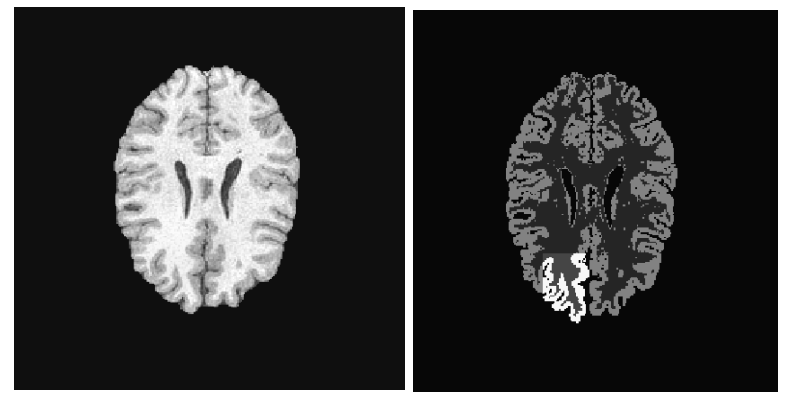

Figure 2. Left: original MRI image; Right: simulated PET phantom generated from the segmented MRI image. The highlighted region of interest (ROI) will be used in the evaluation procedure.

Bias-variance curves are shown in Fig. 4 for all the methods considered. These results indicate that the MESH MAP-W method offers the most accurate quantitative results over a relatively large operating range.

The execution time of the proposed algorithms was similar to our previously reported results [1]. The mesh based methods were approximately an order of magnitude faster than the pixel-based ones.

\section{CONCLUSIONS AND FUTURE WORK}

The preliminary results obtained in this study suggest that the proposed mesh approach is a feasible method for using an anatomical (MR) image to assist in reconstructing a functional (PET) image. In future work we will refine the mesh generation procedure so that the mesh structure is generated jointly from the MR image and a preliminary reconstruction of the PET image. By this procedure it may be possible to further improve image quality, including resolution recovery.

\section{REFERENCES}

[1] J. G. Brankov, Y. Yang, M. V. Narayanan, and M. N. Wernick, "Content-adaptive mesh modeling for tomographic image reconstruction," presented at Fully $3 D$ Image Recon. in Radiol. and Nucl. Med., Pacific Grove, California, USA, 2001.

[2] Y. Wang and O. Lee, "Use of two-dimensional deformable mesh structures for video coding .I. The synthesis problem: mesh-based function approximation and mapping," IEEE Trans. on Circuits Syst. Video Tech., vol. 6, pp. 636 -646, 1996.

[3] D. W. Shattuck, S. R. Sandor-Leahy, K. A. Schaper, D. A. Rottenberg, and R. M. Leahy, "Magnetic resonance image tissue classification using a partial polume model," NeuroImage, vol. 13, pp. 856-876, May 2001.

[4] S. Geman and D. Geman, "Stochastic relaxation, Gibbs distributions, and Bayesian restoration of images," IEEE Trans. on Patt. Anal. Mach. Intell., vol. 6, pp. 721-741, 1984. 
[5] R. Leahy and X. Yan, "Incorporation of anatomical MR data for improved functional imaging with PET," in Information Processing in Medical Imaging, A. C. F. Colchester and D. J. Hawkes, Eds. New York: Springer-Verlag, 1991, pp. 102-120.

[6] C. Bouman and K. Sauer, "A generalized Gaussian image model for edge-preserving MAP estimation," IEEE Trans. Image Prcessing., vol. 2, pp. 296-310, 1993.

[7] G. Gindi, M. Lee, A. Rangarajan, and I. G. Zubal, "Bayesian reconstruction of functional images using anatomical information as priors," IEEE Trans. Med. Imaging, vol. 12, pp. 670 -680, Dec. 1993.

[8] S. Sastry and R. E. Carson, "Multimodality Bayesian algorithm for image reconstruction in positron emission tomography: a tissue composition model," IEEE Trans. Med. Imaging, vol. 16, pp. 750 -761, Dec. 1997.

[9] Y. Yang, M. N. Wernick, and J. G. Brankov, "A Fast algorithm for accurate content-adaptive mesh generation," Proc. of IEEE Intl. Conf. on Image Processing (ICIP), 2001 (in press).

[10] P. J. Green, "Bayesian reconstructions from emission tomography data using a modified EM algorithm," IEEE Trans. Med. Imaging, vol. 9, pp. 84-93, 1990.

[11] A. P. Dempster, N. M. Laird, and D. B. Rubin, "Maximum likelihood from incomplete data via the EM algorithm," J. Roy. Statist. Sect., vol. 39, pp. 1-38, 1977.

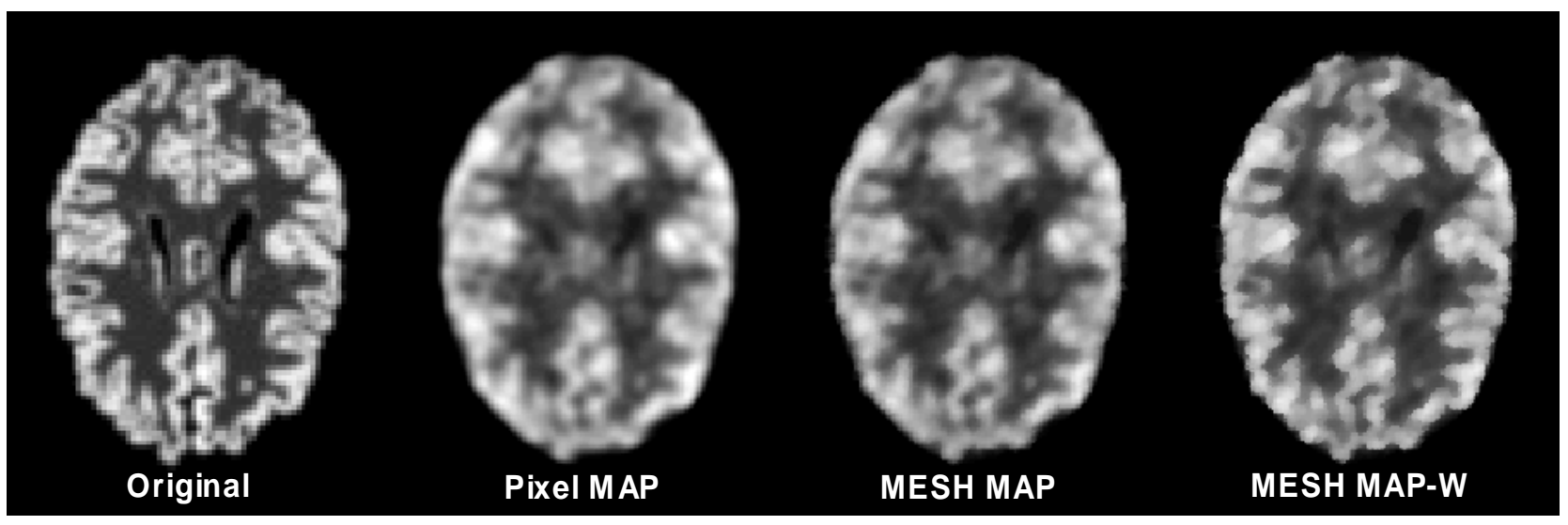

Figure 3. Images obtained by three reconstruction procedures. From left to right: original phantom blurred to intrinsic scanner resolution; pixel MAP reconstruction (EM-OSL/quadratic); MESH MAP reconstruction (EM-OSL/quadratic); MESH MAP-W (EM-OSL/W quadratic) mesh-based reconstruct with varying smoothing parameters. In all algorithms the weighting parameter $\beta$ in (8) was set to 0.005 . The MESH MAP-W image appears to exhibit a degree of resolution recovery not seen in the other images.
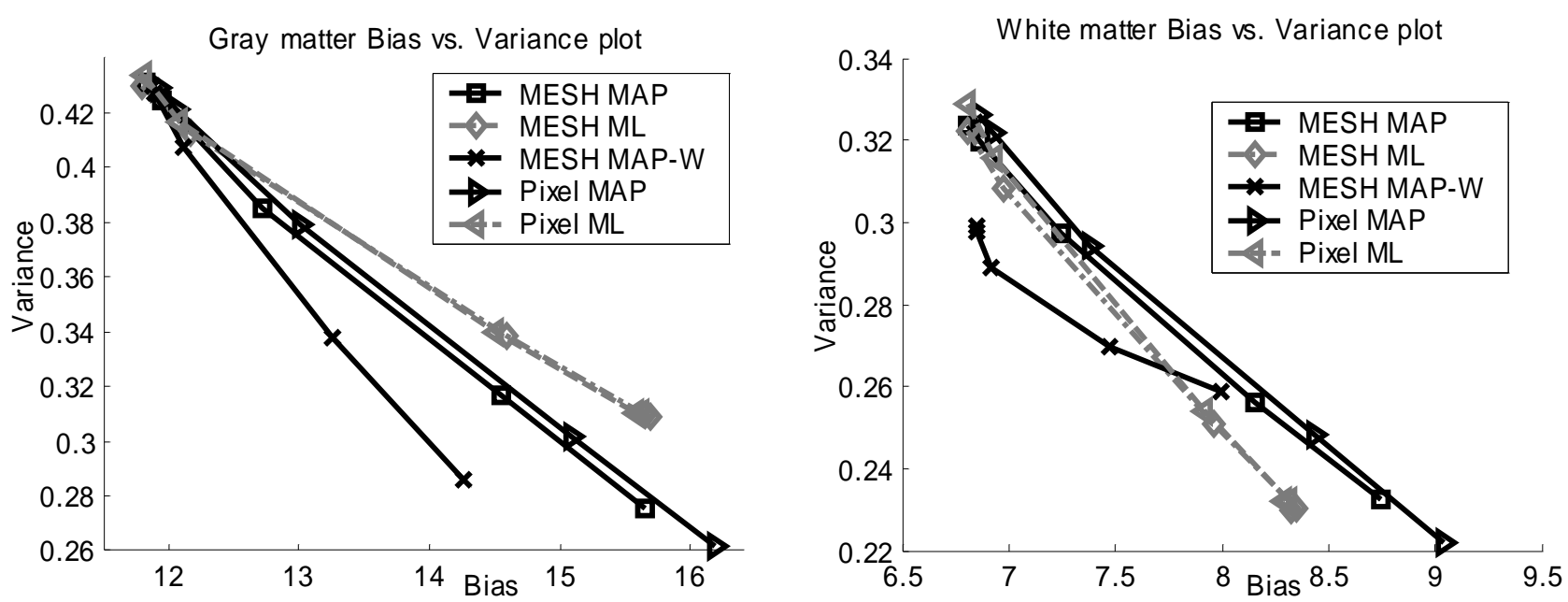

Figure 4. Bias-variance curves, obtained from 56 noise realizations, for the methods considered. The MESH MAP-W algorithm produces the lowest curve for both grey- and white-matter regions, indicating that it produces the best quantitative performance in this preliminary study. 